\section{E-002 DEVELOPMENT OF A PHANTOM THAT CAN MIMIC IDIOPATHIC INTRACRANIAL HYPERTENSION AND TRANSVERSE SINUS STENOSIS}

${ }^{1} \mathrm{~K}$ Valluru, ${ }^{1} \mathrm{~T}$ Shen, ${ }^{1} \mathrm{~K}$ O'Reilly, ${ }^{1} \mathrm{H}$ Haraldsson, ${ }^{1} \mathrm{E}$ Kao, ${ }^{1} \mathrm{~J}$ Leach, ${ }^{1} \mathrm{~A}$ Wright, ${ }^{1} \mathrm{M}$ Ballweber, ${ }^{2} \mathrm{~K}$ Meisel, ${ }^{1} \mathrm{D}$ Saloner, ${ }^{1} \mathrm{M}$ Amans*. 'Radiology and Biomedical Imaging, University of California San Francisco, San Francisco, CA; ${ }^{2}$ Neurology, University of California San Francisco, San Francisco, CA

\subsection{6/neurintsurg-2020-SNIS.38}

Purpose Idiopathic Intracranial Hypertension (IIH) is a pathologic elevation of the intracranial pressure (ICP) with normal CSF composition which mostly affects younger women who are obese. IIH is most commonly presented along with focal stenosis of venous transverse sinus (TS). Venous sinus stenting (VSS) is an effective procedure that can resolve PT in patients with IIH and TS stenosis but has significant relapse rates. A benchtop phantom that can reliably mimic IIH pathophysiology and induce TS stenosis would allow evaluation of factors causing restenosis.

Materials and Methods Our benchtop phantom constitutes an interchangeable $3 \mathrm{D}$ printed TS flow model submerged in a rigid water-filled chamber akin to dura mater. The phantom is equipped with an underwater pressure sensor connected to a microcontroller and capable of continuously relaying the ICP values to a computer in real time. Two calibrated needle gauges connected externally on either side of the TS flow model monitor IVP gradient across the stenosis (figure 1A). ICP is manipulated by infusing additional water analogous to increasing CSF, through the inlet provided on top of the phantom. To better approximate the compliance of the TS in vivo, we tested seven thin-walled flow models of same size made of four different materials with a range of shore hardness factors $(27 \mathrm{~A}-60 \mathrm{~A})$. With glycerol-water mixture pumped through the model, the ICP was increased in the phantom from $0-60 \mathrm{cmH}_{2} \mathrm{O}$ in $5 \mathrm{cmH}_{2} \mathrm{O}$ increments and IVP gradient was recorded across the stenosis $(n=3$ trials per flow model). Measurements were obtained with the phantom positioned to resemble TS in both upright and supine positions respectively.

Results Among all the flow models tested, the model 3D printed with Agilus 30A was found to be a good surrogate for TS when the phantom was oriented in supine position. It produced a stenosis with a mean IVP gradient of $7.84 \mathrm{mmHg}$ at a mean ICP of $25.5 \mathrm{cmH}_{2} \mathrm{O}$, agreeing with typical lumbar puncture opening pressure values $\left(>25 \mathrm{cmH}_{2} \mathrm{O}\right)$ seen in most IIH patients (figure 1B).

Conclusion We developed a benchtop phantom that can mimic the pathophysiology of IIH and can reliably induce TS stenosis by increasing the ICP to the levels typically observed in patients. We anticipate that our phantom will facilitate evaluating anatomical and physiological parameters that induce restenosis and will eventually allow testing of new devices that could minimize VSS relapse rate in IIH patients.

Disclosures K. Valluru: None. T. Shen: None. K. O’Reilly: None. H. Haraldsson: None. E. Kao: None. J. Leach: None. A. Wright: None. M. Ballweber: None. K. Meisel: None. D. Saloner: None. M. Amans: 1; C; NIH R01 HL149124-01A1.

\section{E-003 QUALITY OF LIFE, NEED FOR RETREATMENT, AND THE RE-EQUILIBRATION EFFECT AFTER VENOUS SINUS STENTING FOR IDIOPATHIC INTRACRANIAL HYPERTENSION}

R Garner, J Aldridge, S Wolfe, K Fargen*. Department of Neurosurgery, Wake Forest University, Winston Salem, NC

\subsection{6/neurintsurg-2020-SNIS.39}

Introduction Venous sinus stenting (VSS) is increasingly being used as a treatment for idiopathic intracranial hypertension (IIH). This study aimed to look at long term outcomes and need for further surgical intervention in patients following VSS.

Methods Retrospective analysis was performed on a prospectively-maintained single center database to identify patients with medically refractory IIH who underwent VSS with associated pressure gradient. The senior author performs both VSS and CSF shunting for IIH. Patients with persistent or severe recurrent symptoms after VSS undergo lumbar puncture (LP) to evaluate the effect of VSS on intracranial pressures, therefore need for LP serves as a marker for disease recurrence. Results 81 patients underwent VSS with a mean follow-up of 10 months (median 6 months, range 2 weeks - 38 months). Forty-four (54.3\%) patients underwent repeat LP after VSS due to persistent or recurrent symptoms following the intervention at a mean of 12 months (median 7 months, range
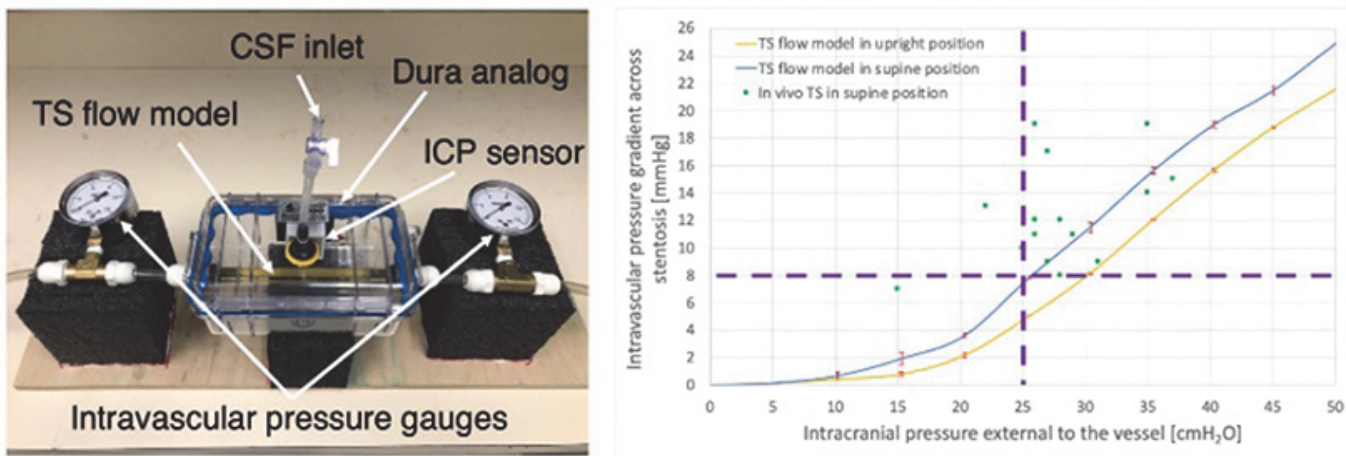

Abstract E-002 Figure 1 A) Benchtop model of Idiopathic Intracranial Hypertension (IIH) and Transverse Sinus (TS) Stenosis with internal components labeled. B) Mean intravascular pressure gradients observed across stenosis under increased intracranial pressure (ICP) using transverse sinus model 3D printed using Agilus 30A $(n=3)$ oriented in upright and supine positions respectively with respect to in vivo measurements. In vivo venous manometry and lumbar puncture measurements were obtained from IIH patients $(n=15)$ lying in supine position who were treated later by venous sinus stenting (VSS) intervention. All the patients had an opening cerebrospinal fluid pressure (CSFp) of $25 \mathrm{~cm} \mathrm{H}_{2} \mathrm{O}$, measured by lumbar puncture and a pressure gradient of $>8 \mathrm{mmHg}$ across the transverse sinus stenosis before the intervention (purple dashed lines) 